\title{
关于双参数量子群的注记 (I)
}

\author{
胡乃红*，裴玉峰 \\ 华东师范大学数学系, 上海 200062 \\ *E-mail: nhhu@math.ecnu.edu.cn \\ 收稿日期: 2007-02-12; 接受日期: 2007-09-03 \\ 国家自然科学基金 (批准号: 10431040, 10728102) 和教育部高校优秀青年教师教学科研奖励计划、教育部博士点基金、 \\ 上海市科委青年科技启明星追踪计划、国家重点学科、教育部长江学若创新团队项目和上海市重点学科等资助项目
}

摘要 借助于 Euler 型, 给出了一类 (对应于半单 Lie 代数的) 双参数量子群的更为简便 的定义方式, 证明了所定义量子群的正部分在双参数满足适当条件下是互为 2-上圈形变的, 并给出了该正部分在 Kashiwara 意义下的斜微分算子实现.

关键词双参数量子群 2 -上圈形变

$\operatorname{MSC}(2000)$ 主题分类 $17 \mathrm{~B} 37,81 \mathrm{R} 50,17 \mathrm{~B} 35$

\section{1 引言}

20 世纪 80 年代中期, 源于求解量子 Yang-Baxter 方程的研究, 俄罗斯数学家 Drinfel'd 和日本数学物理学家 Jimbo 分别提出了量子群的概念. (单参数) 量子群 $U_{q}(\mathfrak{g}$ ) 作为可对称化 Kac-Moody 代数的普遍包络代数形变, 是非交换非余交换的 Hopf 代数. 20 世纪 90 年代初, 有关这些单参数量子群的多参数推广被国际上许多著名专家所探讨, 基本上归结为由以下两 个途径得到: 或者通过定义在某个自由交换群上的 2-上圈来对所阶化的代数结构做乘法的 扭 ${ }^{[1]}$; 或者对余代数结构做 Drinfel'd 意义下的余乘扭 ${ }^{[2,3]}$. 提请读者注意的是, 这种 2 -上圈 扭或者 Drinfel'd 扭所提供的形变, 现在已成为 Hopf 代数或量子群理论中产生新的 (扭) 双代 数或 Hopf 代数的重要方法.

受到定义在偏序集上 (上下行微分算子所满足的双参数型 Serre 关系式) 的上下行代数 (down-up 代数) 的启发 ${ }^{[4]}$, Benkart 和 Witherspoon 等人于本世纪初在系列论文 ${ }^{[5-8]}$ 中分别 研究了一般线性 Lie 代数 $\mathfrak{g l}_{n}$ 和特殊线性 Lie 代数 $\mathfrak{s l}_{n}$ (即 $A$ 型) 的双参数量子群的结构与表 示. 随后, 胡乃红及其合作者 Bergeron 和 $\mathrm{Gao}^{[9,10]}$ 首次给出了 Benkart-Witherspoon 意义下 双参数量子正交群和量子辛群的定义, 探讨了 $A, B, C, D$ 型双参数量子群的 Lusztig 对称性 质, 证明了当对应的单 Lie 代数的秩为 2 时, 双参数量子群到其相伴双参数量子群对象间存 在着 Lusztig 对称性; 而当单 Lie 代数的秩大于 2 时, Lusztig 对称性存在的充要条件是双参数 
必须退化为单参数的情形. 这个定理表明: 双参数量子群与标准的单参数 Drinfeld-Jimbo 型 量子群的本质差别所在 (并直接导致了双参数量子群的结构与表示在研究手段上具有显著的 差异, 如 PBW 基的刻画等问题就需要寻求组合的解决途径). 在双参数的商为非单位根情形 下, 文献 [10] 发展并定型了相应的有限维权模范畴的表示论 (比较文献 [6]).

近年来, 胡乃红及其研究组继续本计划的研究: 胡乃红和师前 ${ }^{[11]}$ 定义了例外型 $G_{2}$ 型 双参数量子群并刻画了其 Lusztig 对称性. 白晓棠和胡乃红 ${ }^{[12]}$ 给出了例外型 $E$ - 系列的定 义结构, 运用 Lyndon 词的组合构造了 $E_{6}$ 的凸性 PBW 基, 计算了其量子 $R$ - 矩阵. 在双参 数均为单位根时, Lusztig “小” 量子群 (即限制型量子群) 结构及其新的 Ribbon 元构造, $B$ 型 被胡乃红和王秀玲得到 ${ }^{[13,14] ;} D$ 型被白晓棠和胡乃红得到 ${ }^{[15]}$; 最近, 王秀玲、陈茹已分别 得到 $F_{4}$ 和 $C$ 型的相应刻画. 有限型双参数量子群到仿射型推广的工作, 仿射 $A_{\ell}^{(1)}$ 型由胡 乃红、Rosso、张红莲合作得到, 文献 [16] 具体构造并证明了这种仿射双参数量子群具有著名 的 “Dinfel'd 实现” 形式 (从而具有 “好的” 表示论), 首次提出 “量子仿射型 Lyndon 基” 的概 念和具体构造 (即使退化到单参数仿射量子群情形, 与 Beck 的 PBW 基相对照, 这种构造法 也更具明晰性); 无扭仿射 $D^{(1)}, E^{(1)}$ 型的相应结果由胡乃红和张红莲 [17] 得到, 文献 [17] 还 构造了无扭仿射 $(A D E)^{(1)}$ 型的双参数顶点表示; 无扭仿射 $(B C F G)^{(1)}$ 型的顶点表示由张红 莲 ${ }^{[18]}$ 进一步得到, 其中无扭仿射型双参数量子群的具有反对合不变性生成函数（内蕴统一) 定义途径为胡乃红在 ICTP 访问期间得到.

本文借助于 Euler 型 (或称 Ringel 型), 给出了对所有半单 Lie 代数都适合的一类双参数 量子群的统一定义. 形式上更为简洁, 但缺点是并不一定适合于定义仿射型 (到目前为止由 此定义的仿射型, 还不能像文献 [16-18] 那样给出我们所期望的 “Drinfel'd 实现” 形式). 值得 注意的是, 这种定义也不能完全涵盖前述已有的有限型情形, 如与文献 [9] 的 $D$ 型情形不一 致. 这个现象表明: 双参数量子群的定义是不唯一的. 这正是本注记所要讨论的一个侧面之 一. 而为弄清定义不唯一性的缘由, 引发了我们对多参数量子群的关注. 事实上, 只有在多参 数背景下, 才可以弄清发生这种不唯一性的定义之间的内在联系 [19].

如文献 $[5,9,11,12,16,17]$ 一样, 本文所定义的量子群也具有 Drinfel'd double 结构和三 角分解结构 (见第 2 节). 作为本注记的一个主要结论, 我们证明了: 在双参数满足适当条件下, 这些量子群作为 $Q^{+}$- 阶化结合代数, 其 “正” 部分是互为 2 -上圈形变的. 这个事实洞悉了我 们所定义的双参数量子群和标准的单参数 Drinfel'd-Jimbo 型量子群的内在联系 (见第 3 节). 第 4 节, 通过分派 $U_{r, s}(\mathfrak{g})$ 的正部分的典范生成元 $e_{i}$ 于 Kashiwara 意义下 ${ }^{[20]}$ 的某些双参数 斜微分算子, 我们给出了其正部分的一个算子实现.

\section{Euler 型和双参数量子群的定义}

设 $C=\left(a_{i j}\right)_{i, j \in I}$ 是有限型 Cartan 矩阵, $\mathfrak{g}$ 为相应的定义在有理数域 $\mathbb{Q}$ 上的半单 Lie 代 数. 设 $\left\{d_{i} \mid i \in I\right\}$ 为一组互素的正整数, 使得 $d_{i} a_{i j}=d_{j} a_{j i}$, 对 $i, j \in I$ 成立. 设 $\mathbb{Q}(r, s)$ 是两 个变量 $r$ 和 $s$ 的有理函数域. 对 $i \in I$, 记 $r_{i}=r^{d_{i}}, s_{i}=s^{d_{i}}$. 设 $\langle\cdot, \cdot\rangle$ 是定义在根格 $Q$ 上的双 线性型—— 称为 Euler 型或 Ringel 型, 其定义如下:

$$
\langle i, j\rangle:=\left\langle\alpha_{i}, \alpha_{j}\right\rangle= \begin{cases}d_{i} a_{i j} & i<j, \\ d_{i} & i=j, \\ 0 & i>j .\end{cases}
$$


定义 2.1 双参数量子群 $U_{r, s}(\mathfrak{g})$ 是定义在域 $\mathbb{Q}(r, s)$ 上的含 1 结合代数, 由生成元 $e_{i}, f_{i}, \omega_{i}^{ \pm 1}, \omega_{i}^{\prime \pm 1}(i \in I)$ 生成, 并满足以下关系式:

$$
\begin{array}{ll}
\omega_{i}^{ \pm 1} \omega_{j}^{ \pm 1}=\omega_{j}^{ \pm 1} \omega_{i}^{ \pm 1}, & \omega_{i}^{\prime \pm 1} \omega_{j}^{\prime \pm 1}=\omega_{j}^{\prime \pm 1} \omega_{i}^{\prime \pm 1}, \\
\omega_{i}^{ \pm 1} \omega_{j}^{\prime \pm 1}=\omega_{j}^{\prime \pm 1} \omega_{i}^{ \pm 1}, & \omega_{i}^{ \pm 1} \omega_{i}^{\mp 1}=\omega_{i}^{\prime \pm 1} \omega_{i}^{\prime \mp 1}=1 . \\
\omega_{i} e_{j} \omega_{i}^{-1}=r^{\langle j, i\rangle} s^{-\langle i, j\rangle} e_{j}, & \omega_{i}^{\prime} e_{j} \omega_{i}^{\prime-1}=r^{-\langle i, j\rangle} s^{\langle j, i\rangle} e_{j} . \\
\omega_{i} f_{j} \omega_{i}^{-1}=r^{-\langle j, i\rangle} s^{\langle i, j\rangle} f_{j}, & \omega_{i}^{\prime} f_{j} \omega_{i}^{\prime-1}=r^{\langle i, j\rangle} s^{-\langle j, i\rangle} f_{j} . \\
e_{i} f_{j}-f_{j} e_{i}=\delta_{i, j} \frac{\omega_{i}-\omega_{i}^{\prime}}{r_{i}-s_{i} .} & \\
\sum_{k=0}^{1-a_{i j}}(-1)^{k}\left(\begin{array}{c}
1-a_{i j} \\
k
\end{array}\right)_{r_{i} s_{i}^{-1}} c_{i j}^{(k)} e_{i}^{1-a_{i j}-k} e_{j} e_{i}^{k}=0, \quad i \neq j, \\
\sum_{k=0}^{1-a_{i j}}(-1)^{k}\left(\begin{array}{c}
1-a_{i j} \\
k
\end{array}\right)_{r_{i} s_{i}^{-1}} c_{i j}^{(k)} f_{i}^{k} f_{j} f_{i}^{1-a_{i j}-k}=0, \quad i \neq j,
\end{array}
$$

其中 $c_{i j}^{(k)}=\left(r_{i} s_{i}^{-1}\right)^{\frac{k(k-1)}{2}} r^{k\langle j, i\rangle} s^{-k\langle i, j\rangle}(i \neq j)$, 且对一个符号 $v$, 采用以下记号:

$$
\begin{aligned}
& (n)_{v}=\frac{v^{n}-1}{v-1}, \quad(n)_{v} !=(1)_{v}(2)_{v} \cdots(n)_{v}, \\
& \left(\begin{array}{l}
n \\
k
\end{array}\right)_{v}=\frac{(n)_{v} !}{(k)_{v} !(n-k)_{v} !}, \quad n \geqslant k \geqslant 0,
\end{aligned}
$$

且 $(0)_{v} !=1$.

代数 $U_{r, s}(\mathfrak{g})$ 有 Hopf 代数结构, 其余乘、余单位和对极定义如下:

$$
\begin{array}{ll}
\Delta\left(\omega_{i}^{ \pm 1}\right)=\omega_{i}^{ \pm 1} \otimes \omega_{i}^{ \pm 1}, & \Delta\left(\omega_{i}^{\prime \pm 1}\right)=\omega_{i}^{\prime \pm 1} \otimes \omega_{i}^{\prime \pm 1}, \\
\Delta\left(e_{i}\right)=e_{i} \otimes 1+\omega_{i} \otimes e_{i}, & \Delta\left(f_{i}\right)=1 \otimes f_{i}+f_{i} \otimes \omega_{i}^{\prime}, \\
\varepsilon\left(\omega_{i}^{ \pm 1}\right)=\varepsilon\left(\omega_{i}^{\prime \pm 1}\right)=1, & \varepsilon\left(e_{i}\right)=\varepsilon\left(f_{i}\right)=0, \\
S\left(\omega_{i}^{ \pm 1}\right)=\omega_{i}^{\mp 1}, & S\left(\omega_{i}^{\prime \pm 1}\right)=\omega_{i}^{\prime \mp 1}, \\
S\left(e_{i}\right)=-\omega_{i}^{-1} e_{i}, & S\left(f_{i}\right)=-f_{i} \omega_{i}^{\prime-1} .
\end{array}
$$

注记 2.2 (i) 设 $r=q, s=q^{-1}$, 则 $U_{q, q^{-1}}(\mathfrak{g})$ 模去由 $\omega_{i}^{\prime}-\omega_{i}^{-1}(i \in I)$ 生成的 Hopf 理想, 同构于 Drinfel'd-Jimbo 型单参数量子群 $U_{q}(\mathfrak{g})$ (参见文献 [19]).

(ii) 设 $r=q^{2}, s=1$, 则 $U_{q^{2}, 1}^{+}$同构于 Ringel 定义的 (非扭的) 一般型 Hall 代数 ${ }^{[20]}$.

(iii) 对于有限 $D$ 型, 上述定义不同于文献 [9] 中定义的代数.

（iv）定义 2.1 也许可以用于定义仿射型的情形, 但是此种定义方式所得到的量子群, 因 目前尚无法给出如文献 [16] 所得到的 “Drinfel'd 实现” 形式, 因而异于文献 [16] 中所定义的 仿射型双参数量子群结构.

设 $U_{r, s}^{+}\left(U_{r, s}^{-}\right)$是由元素 $e_{i}\left(f_{i}\right), i \in I$ 生成的 $U_{r, s}:=U_{r, s}(\mathfrak{g})$ 的子代数, $U^{0}$ 是 $U_{r, s}$ 中 由 $\omega_{i}^{ \pm 1}, \omega_{i}^{\prime \pm 1}(i \in I)$ 生成的子代数. 进一步, 设 $U_{r, s}^{\geqslant 0}\left(U_{r, s}^{\leqslant 0}\right)$ 是 $U_{r, s}$ 中由元素 $e_{i}, \omega_{i}^{ \pm 1}(i \in I)$ $\left(f_{i}, \omega_{i}^{\prime \pm 1}, i \in I\right)$ 生成的子代数. 对每个 $\mu \in Q$ ( $\mathfrak{g}$ 的根格), 定义元素 $\omega_{\mu}$ 和 $\omega_{\mu}^{\prime}$ 如下:

$$
\omega_{\mu}=\prod_{i \in I} \omega_{i}^{\mu_{i}}, \quad \omega_{\mu}^{\prime}=\prod_{i \in I} \omega_{i}^{\prime \mu_{i}}, \quad \mu=\sum_{i \in I} \mu_{i} \alpha_{i} \in Q .
$$

对 $\beta \in Q^{+}$(固定一个正根格), 设

$$
U_{r, s}^{ \pm \beta}=\left\{x \in U_{r, s}^{ \pm} \mid \omega_{\mu} x \omega_{-\mu}=r^{\langle\beta, \mu\rangle} s^{-\langle\mu, \beta\rangle} x, \omega_{\mu}^{\prime} x \omega_{-\mu}^{\prime}=r^{-\langle\mu, \beta\rangle} s^{\langle\beta, \mu\rangle} x, \forall \mu \in Q\right\},
$$


则 $U_{r, s}^{ \pm}=\bigoplus_{\beta \in Q^{+}} U_{r, s}^{ \pm \beta}$ 是 $Q^{+-}$阶化的.

命题 2.3 对任意 $i \in I$, 有 $U_{r, s}(\mathfrak{g})$ 的 $\mathbb{Q}$ - 代数自同构 $\Phi$ 和 $\mathbb{Q}(r, s)$ - 代数反自同构 (或称 对合) $\Psi$, 其定义如下:

$$
\begin{aligned}
& \Phi(r)=s^{-1}, \Phi(s)=r^{-1}, \Phi\left(e_{i}\right)=f_{i}, \Phi\left(f_{i}\right)=r_{i} s_{i} e_{i}, \Phi\left(\omega_{i}\right)=\omega_{i}^{\prime}, \Phi\left(\omega_{i}^{\prime}\right)=\omega_{i} . \\
& \Psi\left(e_{i}\right)=f_{i}, \quad \Psi\left(f_{i}\right)=e_{i}, \quad \Psi\left(\omega_{i}\right)=\omega_{i}, \quad \Psi\left(\omega_{i}^{\prime}\right)=\omega_{i}^{\prime} .
\end{aligned}
$$

证明 直接验证即可.

命题 2.4 存在唯一的双线性斜配对

$$
(\cdot, \cdot): U_{r, s}^{\leqslant 0} \times U_{r, s}^{\geqslant 0} \longrightarrow \mathbb{Q}(r, s),
$$

使得对所有 $x, x^{\prime} \in U_{r, s}^{\geqslant 0}, y, y^{\prime} \in U_{r, s}^{\leqslant 0}, \mu, \nu \in Q, i, j \in I$, 有

$$
\begin{aligned}
& \left(y, x x^{\prime}\right)=\left(\Delta(y), x^{\prime} \otimes x\right), \quad\left(y y^{\prime}, x\right)=\left(y \otimes y^{\prime}, \Delta(x)\right), \\
& \left(f_{i}, e_{j}\right)=\delta_{i, j} \frac{1}{s_{i}-r_{i}}, \quad\left(\omega_{\mu}^{\prime}, \omega_{\nu}\right)=r^{\langle\mu, \nu\rangle} s^{-\langle\nu, \mu\rangle}, \\
& \left(\omega_{\mu}^{\prime}, e_{i}\right)=0, \quad\left(f_{i}, \omega_{\mu}\right)=0 .
\end{aligned}
$$

证明 $U_{r, s}(\mathfrak{g})$ 的余代数结构在 $\left(U_{r, s}^{\geqslant 0}\right)^{*}$ 上定义了如下的代数结构:

$$
\left(\gamma_{1} \gamma_{2}\right)(x):=\left(\gamma_{1} \otimes \gamma_{2}\right)(\Delta(x)), \quad \forall \gamma_{1}, \gamma_{2} \in\left(U_{r, s}^{\geqslant 0}\right)^{*}, \quad x \in U_{r, s}^{\geqslant 0} .
$$

单位元由 $\varepsilon$ 给出. 对任何 $i \in I, \mu \in Q$, 定义线性泛函 $\gamma_{\mu}, \xi_{i} \in\left(U_{r, s}^{\geqslant 0}\right)^{*}$ 如下:

$$
\begin{aligned}
& \gamma_{\mu}\left(x \omega_{\nu}\right)=r^{\langle\mu, \nu\rangle} s^{-\langle\nu, \mu\rangle} \varepsilon(x) \quad\left(\forall x \in U_{r, s}^{+}, \nu \in Q\right), \\
& \xi_{i}\left(x \omega_{\nu}\right)=0 \quad\left(\forall x \in U_{r, s}^{+\beta}, \beta \in Q^{+}-\left\{\alpha_{i}\right\}, \nu \in Q\right), \\
& \xi_{i}\left(e_{i} \omega_{\nu}\right)=\frac{1}{s_{i}-r_{i}} \quad(\forall \nu \in Q) .
\end{aligned}
$$

定义线性映射

$$
\varphi: U_{r, s}^{\leqslant 0} \longrightarrow\left(U_{r, s}^{\geqslant 0}\right)^{*}
$$

在生成元上形如 $\phi\left(\omega_{\mu}^{\prime}\right)=\gamma_{\mu}, \phi\left(f_{i}\right)=\xi_{i}$, 并作代数化扩充定义. 易验证 $\phi$ 是有意义的. 现在 对 $x \in U_{r, s}^{\leqslant 0}, y \in U_{r, s}^{\geqslant 0}$, 定义斜配对

$$
(\cdot, \cdot): U_{r, s}^{\leqslant 0} \times U_{r, s}^{\geqslant 0} \longrightarrow \mathbb{Q}(r, s)
$$

为 $(x, y):=\phi(x)(y)$. 约束条件

$$
\left(y y^{\prime}, x\right)=\left(y \otimes y^{\prime}, \Delta(x)\right), \quad\left(y, x x^{\prime}\right)=\left(\Delta(y), x^{\prime} \otimes x\right),
$$

对 $x \in U_{r, s}^{\geqslant 0}, y, y^{\prime} \in U_{r, s}^{\leqslant 0}$ 成立, 可以归纳地加以证明. 其余条件成立是明显的. 而且, 双线性 型 $(\cdot, \cdot)$ 被唯一确定也是清楚的. 证明完毕.

基于命题 2.4 , 类似于文献 [9] 的定理 2.5 的证明, 我们有

推论 2.5 关于斜配对 $(\cdot, \cdot), U_{r, s}(\mathfrak{g})$ 可以实现为其 Hopf 子代数 $U_{r, s}^{\geqslant 0}$ 和 $U_{r, s}^{\geqslant 0}$ 的一个 Drinfel'd double 结构, 即

$$
U_{r, s}(\mathfrak{g}) \cong \mathcal{D}\left(U_{r, s}^{\geqslant 0}, U_{r, s}^{\leqslant 0}\right) .
$$

作为该 Drinfel'd double 结构的一个直接推论, 与文献 [9] 中推论 2.9 的推理一样, 有

推论 $2.6 U_{r, s}(\mathfrak{g})$ 有标准三角分解式

$$
U_{r, s}(\mathfrak{g}) \cong U_{r, s}^{-} \otimes U_{r, s}^{0} \otimes U_{r, s}^{+},
$$


其中 $U_{r, s}^{0}=\bigoplus_{\mu, \nu \in Q} \mathbb{Q}(r, s) \omega_{\nu}^{\prime} \omega_{\mu}, U_{r, s}^{ \pm}=\bigoplus_{\beta \in Q+} U_{r, s}^{ \pm \beta}$.

\section{3 “正部分" $U_{r, s}^{+}$的 $2-$ 上圈形变}

引理 3.1 ${ }^{[1]}$ 设 $A=\bigoplus_{g \in G} A_{g}$ 是域 $k$ 上 $G$ - 阶化结合代数, 其中 $G$ 是 Abel 群. 设 $\psi$ : $G \times G \rightarrow k^{*}$ 是群 $G$ 的一个 2-上圈. 在 $A$ 上引进一个新的乘法 $*$ 如下: 对任何 $x \in A_{g}, y \in A_{h}$, 其中 $g, h \in G$, 定义

$$
x * y=\psi(g, h) x y .
$$

记此新代数为 $A^{\psi}$, 则 $A^{\psi}$ 是一个 $G$ - 阶化结合代数 (因为 $\psi$ 是 2-上圈). 代数 $A^{\psi}$ 称为代数 $A$ 通过上圈 $\psi$ 的一个形变.

本节取参数 $r, s, r^{\prime}, s^{\prime} \in \mathcal{C}^{*}$, 并考虑两个定义在复数域 $\mathcal{C}$ 上的代数 $U_{r, s}^{+}$和 $U_{r^{\prime}, s^{\prime}}^{+}$. 注意到, 它们都是 $Q$ - 阶化的. 鉴于引理 3.1, 以下主要结论的证明是有趣的.

定理 3.2 如果 $r s^{-1}=r^{\prime} s^{-1}$ 或 $r s^{-1}=r^{\prime-1} s^{\prime}$, 那么 $U_{r, s}^{+}$和 $U_{r^{\prime}, s^{\prime}}^{+}$是互为 2 -上圈形变的 代数结构.

证明 (I) 假设 $r s^{-1}=r^{\prime} s^{\prime-1}$. 在此情形, 在 $U_{r, s}^{+}$上定义一个新的乘积 $*$ 如下:

$$
x * y=\psi(\mu, \nu) x y=\left(r^{-1} r^{\prime}\right)^{\langle\mu, \nu\rangle} x y,
$$

对任意 $x \in U_{r, s}^{+\mu}, y \in U_{r, s}^{+\nu}$, 其中 $\psi: Q \times Q \longrightarrow \mathcal{C}^{*}$, 使得 $\psi(\mu, \nu)=\left(r^{-1} r^{\prime}\right)^{\langle\mu, \nu\rangle}$.

注意到, $\psi$ 是 $Q \times Q$ 上的一个双特征标, 明显地, 这是 Abel 群 $Q$ 上的一个 2-上圈. 这个 事实确保了新的乘积是结合的.

下面只要证明以下的关系式即可:

$$
\begin{array}{ll}
\sum_{k=0}^{1-a_{i j}}(-1)^{k}\left(\begin{array}{c}
1-a_{i j} \\
k
\end{array}\right)_{r_{i} s_{i}^{-1}} c_{i j}^{(k)} e_{i}^{*\left(1-a_{i j}-k\right)} * e_{j} * e_{i}^{* k}=0, & i \neq j ; \\
\sum_{k=0}^{1-a_{i j}}(-1)^{k}\left(\begin{array}{c}
1-a_{i j} \\
k
\end{array}\right)_{r_{i} s_{i}^{-1}} c_{i j}^{(k)} f_{i}^{* k} * f_{j} * f_{i}^{*\left(1-a_{i j}-k\right)}=0, & i \neq j,
\end{array}
$$

其中

$$
c_{i j}^{(k)}=\left(r_{i} s_{i}^{-1}\right)^{\frac{k(k-1)}{2}} r^{k\langle j, i\rangle} s^{-k\langle i, j\rangle}, \quad i \neq j .
$$

由 $*$ - 乘积的定义有

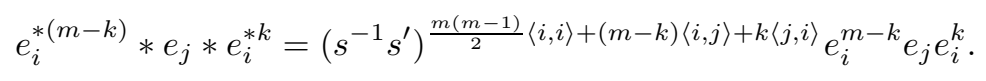

情形 $1 . i<j$, 即 $\langle j, i\rangle=0$ : 当 $m=1-a_{i j}$ 时, 有

$$
\begin{aligned}
& \sum_{k=0}^{m}(-1)^{k}\left(\begin{array}{c}
m \\
k
\end{array}\right)_{r_{i} s_{i}^{-1}} c_{i j}^{(k)} e_{i}^{*(m-k)} * e_{j} * e_{i}^{* k}
\end{aligned}
$$

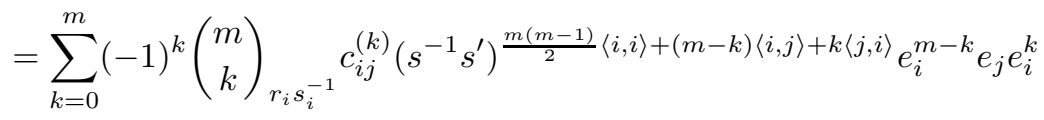

$$
\begin{aligned}
& =\sum_{k=0}^{m}(-1)^{k}\left(\begin{array}{c}
m \\
k
\end{array}\right)_{r_{i} s_{i}^{-1}}\left(r_{i} s_{i}^{-1}\right)^{\frac{k(k-1)}{2}} s^{-k\langle i, j\rangle}\left(s^{-1} s^{\prime}\right)^{\frac{m(m-1)}{2} d_{i}+(m-k) d_{i} a_{i j}} e_{i}^{m-k} e_{j} e_{i}^{k}
\end{aligned}
$$




$$
\begin{aligned}
& =\left(s_{i}^{-1} s_{i}^{\prime}\right)^{\frac{m(m-1)}{2}+m a_{i j}} \sum_{k=0}^{m}(-1)^{k}\left(\begin{array}{c}
m \\
k
\end{array}\right)_{r_{i} s_{i}^{-1}}\left(r_{i} s_{i}^{-1}\right)^{\frac{k(k-1)}{2}} s_{i}^{-k a_{i j}}\left(s_{i} s_{i}^{\prime-1}\right)^{k a_{i j}} e_{i}^{m-k} e_{j} e_{i}^{k} \\
& =\left(s_{i}^{-1} s_{i}^{\prime}\right)^{\frac{m(m-1)}{2}}+m a_{i j} \sum_{k=0}^{m}(-1)^{k}\left(\begin{array}{c}
m \\
k
\end{array}\right)_{r_{i} s_{i}^{-1}}\left(r_{i} s_{i}^{-1}\right)^{\frac{k(k-1)}{2}}\left(s_{i}^{\prime-1}\right)^{k a_{i j}} e_{i}^{m-k} e_{j} e_{i}^{k} \\
& =\left(s_{i} s_{i}^{\prime-1}\right)^{\frac{m(m-1)}{2}} \sum_{k=0}^{m}(-1)^{k}\left(\begin{array}{c}
m \\
k
\end{array}\right)_{r_{i}^{\prime} s_{i}^{\prime-1}}\left(r_{i}^{\prime} s_{i}^{\prime-1}\right)^{\frac{k(k-1)}{2}} r^{\prime k\langle j, i\rangle} s^{-k\langle i, j\rangle} e_{i}^{m-k} e_{j} e_{i}^{k} \\
& =\left(s_{i} s_{i}^{\prime-1}\right)^{\frac{m(m-1)}{2}} \underbrace{\sum_{k=0}^{m}(-1)^{k}\left(\begin{array}{c}
m \\
k
\end{array}\right)_{r_{i}^{\prime} s_{i}^{\prime-1}} c_{i j}^{\prime(k)} e_{i}^{m-k} e_{j} e_{i}^{k}} \\
& =0 \quad\left(U_{r^{\prime}, s^{\prime}}^{+} \text {中的 }\left(r^{\prime}, s^{\prime}\right)\right. \text {-Serre关系式). }
\end{aligned}
$$

情形 $2 . i>j$, 即 $\langle i, j\rangle=0$ : 当 $m=1-a_{i j}$ 时, 有

$$
\begin{aligned}
\sum_{k=0}^{m} & (-1)^{k}\left(\begin{array}{c}
m \\
k
\end{array}\right)_{r_{i} s_{i}^{-1}} c_{i j}^{(k)} e_{i}^{*(m-k)} * e_{j} * e_{i}^{* k} \\
& =\sum_{k=0}^{m}(-1)^{k}\left(\begin{array}{c}
m \\
k
\end{array}\right)_{r_{i} s_{i}^{-1}} c_{i j}^{(k)}\left(s^{-1} s^{\prime}\right)^{\frac{m(m-1)}{2}\langle i, i\rangle+(m-k)\langle i, j\rangle+k\langle j, i\rangle} e_{i}^{m-k} e_{j} e_{i}^{k} \\
& =\sum_{k=0}^{m}(-1)^{k}\left(\begin{array}{c}
m \\
k
\end{array}\right)_{r_{i} s_{i}^{-1}}\left(r_{i} s_{i}^{-1}\right)^{\frac{k(k-1)}{2}} r_{i}^{k a_{i j}}\left(s^{-1} s^{\prime}\right)^{\frac{m(m-1)}{2}} d_{i}+k d_{i} a_{i j} e_{i}^{m-k} e_{j} e_{i}^{k} \\
& =\left(s_{i}^{-1} s_{i}^{\prime}\right)^{\frac{m(m-1)}{2}} \sum_{k=0}^{m}(-1)^{k}\left(\begin{array}{c}
m \\
k
\end{array}\right)_{r_{i} s_{i}^{-1}}\left(r_{i} s_{i}^{-1}\right)^{\frac{k(k-1)}{2}} r_{i}^{k a_{i j}}\left(s_{i}^{-1} s_{i}^{\prime}\right)^{k a_{i j}} e_{i}^{m-k} e_{j} e_{i}^{k} \\
& =\left(s_{i}^{-1} s_{i}^{\prime}\right)^{\frac{m(m-1)}{2}} \sum_{k=0}^{m}(-1)^{k}\left(\begin{array}{c}
m \\
k
\end{array}\right)_{r_{i} s_{i}^{-1}}\left(r_{i} s_{i}^{-1}\right)^{\frac{k(k-1)}{2}}\left(r_{i}^{\prime}\right)^{k a_{i j}} e_{i}^{m-k} e_{j} e_{i}^{k} \\
& =\left(s_{i}^{-1} s_{i}^{\prime}\right)^{\frac{m(m-1)}{2}} \sum_{k=0}^{m}(-1)^{k}\left(\begin{array}{c}
m \\
k
\end{array}\right)_{r_{i}^{\prime} s_{i}^{\prime-1}}\left(r_{i}^{\prime} s_{i}^{\prime-1}\right)^{\frac{k(k-1)}{2}} r^{\prime k\langle j, i\rangle} s^{\prime-k\langle i, j\rangle} e_{i}^{m-k} e_{j} e_{i}^{k} \\
& =\left(s_{i}^{-1} s_{i}^{\prime}\right)^{\frac{m(m-1)}{2}} \sum_{k=0}^{m}(-1)^{k}\left(\begin{array}{c}
m \\
k
\end{array}\right)_{r_{i}^{\prime} s_{i}^{\prime-1}} c_{i j}^{\prime(k)} e_{i}^{m-k} e_{j} e_{i}^{k}=0 .
\end{aligned}
$$

因此, $U_{r, s}^{+}$和 $U_{r^{\prime}, s^{\prime}}^{+}$是互为 2 -上圈形变的.

(II) 假设 $r s^{-1}=r^{\prime-1} s^{\prime}$. 在此情形, 我们可在 $U_{r, s}^{+}$上定义另一个新的乘积 $*$ 如下:

$$
x * y=\psi(\mu, \nu) x y=\left(r^{\prime} s^{-1}\right)^{\langle\mu, \nu\rangle} x y,
$$

对任意 $x \in U_{r, s}^{+\mu}, y \in U_{r, s}^{+\nu}$, 其中 $\psi: Q \times Q \longrightarrow \mathcal{C}^{*}$, 使得 $\psi(\mu, \nu)=\left(r^{\prime} s^{-1}\right)^{\langle\mu, \nu\rangle}$. 于是有下面的 推导:

情形 $1^{\prime}$. $i<j$, 即 $\langle j, i\rangle=0$ : 当 $m=1-a_{i j}$ 时, 有

$$
\begin{aligned}
& \sum_{k=0}^{m}(-1)^{k}\left(\begin{array}{c}
m \\
k
\end{array}\right)_{r_{i} s_{i}^{-1}} c_{i j}^{(k)} e_{i}^{*(m-k)} * e_{j} * e_{i}^{* k} \\
& =\sum_{k=0}^{m}(-1)^{k}\left(\begin{array}{c}
m \\
k
\end{array}\right)_{r_{i} s_{i}^{-1}} c_{i j}^{(k)}\left(r^{\prime} s^{-1}\right)^{\frac{m(m-1)}{2}\langle i, i\rangle+(m-k)\langle i, j\rangle+k\langle j, i\rangle} e_{i}^{m-k} e_{j} e_{i}^{k}
\end{aligned}
$$




$$
\begin{aligned}
& =\sum_{k=0}^{m}(-1)^{k}\left(\begin{array}{c}
m \\
k
\end{array}\right)_{r_{i} s_{i}^{-1}}\left(r_{i} s_{i}^{-1}\right)^{\frac{k(k-1)}{2}} s^{-k\langle i, j\rangle}\left(r^{\prime} s^{-1}\right)^{\frac{m(m-1)}{2}} d_{i}+(m-k) d_{i} a_{i j} e_{i}^{m-k} e_{j} e_{i}^{k} \\
& =\left(r_{i}^{\prime} s_{i}^{-1}\right)^{\frac{m(m-1)}{2}}+m a_{i j} \sum_{k=0}^{m}(-1)^{k}\left(\begin{array}{c}
m \\
k
\end{array}\right)_{r_{i} s_{i}^{-1}}\left(r_{i} s_{i}^{-1}\right)^{\frac{k(k-1)}{2}} s_{i}^{-k a_{i j}}\left(s_{i} r_{i}^{\prime-1}\right)^{k a_{i j}} e_{i}^{m-k} e_{j} e_{i}^{k} \\
& =\left(r_{i}^{\prime-1} s_{i}\right)^{\frac{m(m-1)}{2}} \sum_{k=0}^{m}(-1)^{k}\left(\begin{array}{c}
m \\
k
\end{array}\right)_{r_{i}^{\prime-1} s_{i}^{\prime}}\left(r_{i}^{\prime-1} s_{i}^{\prime}\right)^{\frac{k(k-1)}{2}}\left(r_{i}^{\prime-1}\right)^{k a_{i j}} e_{i}^{m-k} e_{j} e_{i}^{k} \\
& =\left(r_{i}^{\prime-1} s_{i}\right)^{\frac{m(m-1)}{2}} \sum_{k=0}^{m}(-1)^{k}\left(\begin{array}{c}
m \\
k
\end{array}\right)_{r_{i}^{\prime} s_{i}^{\prime-1}}\left(r_{i}^{\prime} s_{i}^{\prime-1}\right)^{k^{2}-k m}\left(r_{i}^{\prime-1} s_{i}^{\prime}\right)^{\frac{k(k-1)}{2}} r_{i}^{\prime-k a_{i j}} e_{i}^{m-k} e_{j} e_{i}^{k} \\
& =\left(r_{i}^{\prime-1} s_{i}\right)^{\frac{m(m-1)}{2}} \sum_{k=0}^{m}(-1)^{k}\left(\begin{array}{c}
m \\
k
\end{array}\right)_{r_{i}^{\prime} s_{i}^{\prime-1}}\left(r_{i}^{\prime} s_{i}^{\prime-1}\right)^{\frac{k(k-1)}{2}} r^{\prime k\langle j, i\rangle} s^{\prime-k\langle i, j\rangle} e_{i}^{m-k} e_{j} e_{i}^{k} \\
& =0 .
\end{aligned}
$$

情形 $2^{\prime}$. $i>j$, 即 $\langle i, j\rangle=0$ : 当 $m=1-a_{i j}$ 时, 有

$$
\begin{aligned}
& \sum_{k=0}^{m}(-1)^{k}\left(\begin{array}{c}
m \\
k
\end{array}\right)_{r_{i} s_{i}^{-1}} c_{i j}^{(k)} e_{i}^{*(m-k)} * e_{j} * e_{i}^{* k}
\end{aligned}
$$

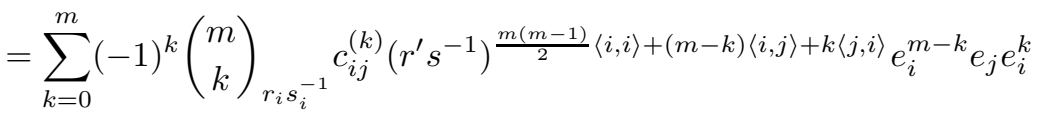

$$
\begin{aligned}
& =\sum_{k=0}^{m}(-1)^{k}\left(\begin{array}{c}
m \\
k
\end{array}\right)_{r_{i} s_{i}^{-1}}\left(r_{i} s_{i}^{-1}\right)^{\frac{k(k-1)}{2}} r_{i}^{k a_{i j}}\left(r^{-1} s^{\prime}\right)^{\frac{m(m-1)}{2} d_{i}+k d_{i} a_{i j}} e_{i}^{m-k} e_{j} e_{i}^{k} \\
& =\left(r_{i}^{-1} s_{i}^{\prime}\right)^{\frac{m(m-1)}{2}} \sum_{k=0}^{m}(-1)^{k}\left(\begin{array}{c}
m \\
k
\end{array}\right)_{r_{i} s_{i}^{-1}}\left(r_{i} s_{i}^{-1}\right)^{\frac{k(k-1)}{2}} r_{i}^{k a_{i j}}\left(r_{i}^{-1} s_{i}^{\prime}\right)^{k a_{i j}} e_{i}^{m-k} e_{j} e_{i}^{k} \\
& =\left(r_{i}^{-1} s_{i}^{\prime}\right)^{\frac{m(m-1)}{2}} \sum_{k=0}^{m}(-1)^{k}\left(\begin{array}{c}
m \\
k
\end{array}\right)_{r_{i}^{\prime-1} s_{i}^{\prime}}\left(r_{i}^{\prime-1} s_{i}^{\prime}\right)^{\frac{k(k-1)}{2}}\left(s_{i}^{\prime}\right)^{k a_{i j}} e_{i}^{m-k} e_{j} e_{i}^{k} \\
& =\left(r_{i}^{-1} s_{i}^{\prime}\right)^{\frac{m(m-1)}{2}} \sum_{k=0}^{m}(-1)^{k}\left(\begin{array}{c}
m \\
k
\end{array}\right)_{r_{i}^{\prime} s_{i}^{\prime-1}}\left(r_{i}^{\prime} s_{i}^{\prime-1}\right)^{k^{2}-k m}\left(r_{i}^{\prime-1} s_{i}^{\prime}\right)^{\frac{k(k-1)}{2}}\left(s_{i}^{\prime}\right)^{k a_{i j}} e_{i}^{m-k} e_{j} e_{i}^{k} \\
& =\left(r_{i}^{-1} s_{i}^{\prime}\right)^{\frac{m(m-1)}{2}} \sum_{k=0}^{m}(-1)^{k}\left(\begin{array}{c}
m \\
k
\end{array}\right)_{r_{i}^{\prime} s_{i}^{\prime-1}}\left(r_{i}^{\prime} s_{i}^{\prime-1}\right)^{\frac{k(k-1)}{2}} r^{\prime k\langle j, i\rangle} s^{-k\langle i, j\rangle} e_{i}^{m-k} e_{j} e_{i}^{k} \\
& =0 .
\end{aligned}
$$

因此, $U_{r, s}^{+}$和 $U_{r^{\prime}, s^{\prime}}^{+}$是互为 2-上圈形变的. 证明完毕.

推论 3.3 (i) $U_{r, s}^{+}$和 $U_{s^{-1}, r^{-1}}^{+}$(即文献 [9] 中所称谓的前一个量子群的相伴对象) 是互 为 2 -上圈形变的. 而且, $U_{r, s}^{+}=U_{s^{-1}, r^{-1}}^{+}$当且仅当 $r s=1$.

(ii) 特别地, 如果 $r s^{-1}=q^{2}$, 那么 $U_{r, s}^{+}, U_{q^{2}, 1}^{+}$和 $U_{q, q^{-1}}^{+}$也是互为 2-上圈形变的.

\section{Kashiwara 斜微分算子与 “正部分” 的实现}

下面的结果源自单参数情形下 Kashiwara 相应工作 ${ }^{[16]}$ 的启发.

命题 4.1 对 $P \in U_{r, s}^{+}$, 存在唯一的 $L, R \in U_{r, s}^{+}$满足方程

$$
\left[P, f_{i}\right]=\frac{\omega_{i} L-\omega_{i}^{\prime} R}{r_{i}-s_{i}},
$$


其中定义 $\partial_{i}(P)=L, \partial_{i}^{\prime}(P)=R$.

证明 假设

$$
\frac{\omega_{i} L_{1}-\omega_{i}^{\prime} R_{1}}{r_{i}-s_{i}}=\frac{\omega_{i} L_{2}-\omega_{i}^{\prime} R_{2}}{r_{i}-s_{i}}
$$

则有

$$
\omega_{i}\left(L_{1}-L_{2}\right)-\omega_{i}^{\prime}\left(R_{1}-R_{2}\right)=0 .
$$

使用推论 2.6 中 $U_{r, s}(\mathfrak{g})$ 的三角分解式, 得到 $L_{1}=L_{2}, R_{1}=R_{2}$. 这意味着 $L$ 和 $R$ 的唯一性 是清楚的.

为证 $L$ 和 $R$ 的存在性, 考虑其阶化分解: $U_{r, s}^{+}=\bigoplus_{\nu \in Q^{+}} U_{r, s}^{+\nu}$. 我们对权的高度使用归纳 法证明这个结论, 即 $\operatorname{ht}(\nu)=\sum m_{i}$, 对 $\nu=\sum m_{i} \alpha_{i}\left(m_{i} \in \mathbb{Z}_{\geqslant 0}\right)$. 如果 $\operatorname{ht}(\nu)=1, P=e_{j}$, 则选 择 $L=R=\delta_{i, j}$. 假设对 $P \in U_{r, s}^{+\nu}$, 存在 $L$ 和 $R$ 满足

则对 $e_{j} P \in U_{r, s}^{+(\nu+1)}$ 有 $h t\left(e_{j} P\right)=\nu+1$, 用归纳假设可得

$$
\left[P, f_{i}\right]=\frac{\omega_{i} L-\omega_{i}^{\prime} R}{r_{i}-s_{i}},
$$

$$
\begin{aligned}
{\left[e_{j} P, f_{i}\right] } & =e_{j}\left[P, f_{i}\right]+\left[e_{j}, f_{i}\right] P \\
& =\frac{1}{r_{i}-s_{i}}\left\{\omega_{i}\left(r^{-\langle j, i\rangle} s^{\langle i, j\rangle} e_{j} L+\delta_{i, j} P\right)-\omega_{i}^{\prime}\left(r^{\langle i, j\rangle} s^{-\langle j, i\rangle} e_{j} R+\delta_{i, j} P\right)\right\} \\
& =\frac{1}{r_{i}-s_{i}}\left\{\omega_{i}\left(r^{-\langle j, i\rangle} s^{\langle i, j\rangle} e_{j} \partial_{i}(P)+\delta_{i, j} P\right)-\omega_{i}^{\prime}\left(r^{\langle i, j\rangle} s^{-\langle j, i\rangle} e_{j} \partial_{i}^{\prime}(P)+\delta_{i, j} P\right)\right\},
\end{aligned}
$$

特别地, 我们得到

$$
\begin{aligned}
& \partial_{i}\left(e_{j} P\right)=r^{-\langle j, i\rangle} s^{\langle i, j\rangle} e_{j} \partial_{i}(P)+\delta_{i, j} P \\
& \partial_{i}^{\prime}\left(e_{j} P\right)=r^{\langle i, j\rangle} s^{-\langle j, i\rangle} e_{j} \partial_{i}^{\prime}(P)+\delta_{i, j} P .
\end{aligned}
$$

引理证毕.

由 $(*)$ 式, 容易得到

$$
\partial_{i}\left(e_{i}^{m}\right)=(m)_{r_{i}^{-1} s_{i}} e_{i}^{m-1}, \quad \partial_{i}^{\prime}\left(e_{i}^{m}\right)=(m)_{r_{i} s_{i}^{-1}} e_{i}^{m-1} .
$$

对 $i \in I$, 引进算子 $E_{i}: U_{r, s}^{+} \longrightarrow U_{r, s}^{+}$, 其定义如下:

$$
E_{i} x=e_{i} x, \quad \forall x \in U_{r, s}^{+} .
$$

于是下面的引理可利用命题 4.1 并归纳地加以证明.

引理 4.2 对 $m \in \mathcal{Z}_{+}, i, j \in I$, 下面的换位关系成立:

$$
\begin{aligned}
& \partial_{i} \partial_{j}^{\prime}=r^{\langle j, i\rangle} s^{-\langle i, j\rangle} \partial_{j}^{\prime} \partial_{i}, \\
& \partial_{i}^{m} E_{j}=r^{-m\langle j, i\rangle} s^{m\langle i, j\rangle} E_{j} \partial_{i}^{m}+\delta_{i, j}(m)_{r_{i}^{-1} s_{i}} \partial_{i}^{m-1}, \\
& \partial_{i}^{\prime m} E_{j}=r^{m\langle i, j\rangle} s^{-m\langle j, i\rangle} E_{j} \partial_{i}^{\prime m}+\delta_{i, j}(m)_{r_{i} s_{i}^{-1}} \partial_{i}^{\prime m-1} .
\end{aligned}
$$

定理 4.3 对任意 $i \neq j \in I$, 有

(i)

$$
\sum_{k=0}^{1-a_{i j}}(-1)^{k}\left(\begin{array}{c}
1-a_{i j} \\
k
\end{array}\right)_{r_{i} s_{i}^{-1}} c_{i j}^{(k)} \partial_{i}^{k} \partial_{j} \partial_{i}^{1-a_{i j}-k}=0,
$$

$$
\sum_{k=0}^{1-a_{i j}}(-1)^{k}\left(\begin{array}{c}
1-a_{i j} \\
k
\end{array}\right)_{r_{i} s_{i}^{-1}} c_{i j}^{(k)} \partial_{i}^{\prime k} \partial_{j}^{\prime} \partial_{i}^{\prime 1-a_{i j}-k}=0 .
$$


于是通过分别指派 $U_{r, s}^{+}$的生成元 $e_{i}$ 对应于 Kashiwara 意义下的斜微分算子 $\partial_{i}$ 或者 $\partial_{i}^{\prime}$, 它们 给出了 $U_{r, s}^{+}$的两个算子实现.

证明 (i) 对任意 $u \in U_{r, s}^{+\mu}$, 我们将对 $\operatorname{ht}(u)=\mu$ 利用归纳法, 证明下面的公式:

$$
\sum_{k=0}^{1-a_{i j}}(-1)^{k}\left(\begin{array}{c}
1-a_{i j} \\
k
\end{array}\right)_{r_{i} s_{i}^{-1}} c_{i j}^{(k)} \partial_{i}^{1-a_{i j}-k} \partial_{j} \partial_{i}^{k} u=0 .
$$

对任意 $v \in U_{r, s}^{+\nu}$ 有 $\operatorname{ht}(v)=\nu<\operatorname{ht}(u)=\mu$, 假设 $(* *)$ 式成立. 记 $u$ 为 $u=e_{\ell} v=E_{\ell} v$, 对某 $\ell \in I$ 和 $v \in U_{r, s}^{+\nu}$. 现在令 $m=1-a_{i j}$.

首先, 注意到

$$
\begin{aligned}
\partial_{i}^{m-k} & \partial_{j} \partial_{i}^{k} E_{\ell} \\
= & \partial_{i}^{m-k} \partial_{j}\left\{r^{-k\langle\ell, i\rangle} s^{k\langle i, \ell\rangle} E_{\ell} \partial_{i}^{k}+\delta_{i, \ell}(k)_{r_{i}^{-1} s_{i}} \partial_{i}^{k-1}\right\} \\
= & \partial_{i}^{m-k}\left\{r^{-k\langle\ell, i\rangle} s^{k\langle i, \ell\rangle}\left(r^{-\langle\ell, j\rangle} s^{\langle j, \ell\rangle} E_{\ell} \partial_{j}+\delta_{\ell, j}\right) \partial_{i}^{k}+\delta_{i, \ell}(k)_{r_{i}^{-1} s_{i}} \partial_{j} \partial_{i}^{k-1}\right\} \\
= & r^{-k\langle\ell, i\rangle} s^{k\langle i, \ell\rangle} r^{-\langle\ell, j\rangle} s^{\langle j, \ell\rangle} \\
& \times\left\{r^{(k-m)\langle\ell, i\rangle} s^{(m-k)\langle i, \ell\rangle} E_{\ell} \partial_{i}^{m-k}+\delta_{\ell, i}(m-k)_{r_{i}^{-1}} s_{i} \partial_{i}^{m-k-1}\right\} \partial_{j} \partial_{i}^{k} \\
& +\delta_{\ell, j} r^{-k\langle\ell, i\rangle} s^{k\langle i, \ell\rangle} \partial_{i}{ }^{m-k} \partial_{i}^{k}+\delta_{i, \ell}(k)_{r_{i}^{-1} s_{i}} \partial_{i}^{m-k} \partial_{j} \partial_{i}^{k-1} \\
= & r^{-m\langle\ell, i\rangle} s^{m\langle i, \ell\rangle} r^{-\langle\ell, j\rangle} s^{\langle j, \ell\rangle} E_{\ell} \partial_{i}^{m-k} \partial_{j} \partial_{i}^{k} \\
& +\delta_{\ell, i} r^{-k\langle\ell, i\rangle} s^{k\langle i, \ell\rangle} r^{-\langle\ell, j\rangle} s^{\langle j, \ell\rangle}(m-k)_{r_{i}^{-1} s_{i}} \partial_{i}^{m-k-1} \partial_{j} \partial_{i}^{k} \\
& +\delta_{i, \ell}(k)_{r_{i}^{-1} s_{i}} \partial_{i}^{m-k} \partial_{j} \partial_{i}^{k-1}+\delta_{\ell, j} r^{-k\langle\ell, i\rangle} s^{k\langle i, \ell\rangle} \partial_{i}{ }^{m},
\end{aligned}
$$

因而得到

$$
\begin{aligned}
& \sum_{k=0}^{m}(-1)^{k}\left(\begin{array}{c}
m \\
k
\end{array}\right)_{r_{i} s_{i}^{-1}} c_{i j}^{(k)} \partial_{i}^{k} \partial_{j} \partial_{i}^{m-k} E_{\ell} \\
&=r^{-m\langle\ell, i\rangle} s^{m\langle i, \ell\rangle} r^{-\langle\ell, j\rangle} s^{\langle j, \ell\rangle} E_{\ell} \sum_{k=0}^{m}(-1)^{k}\left(\begin{array}{c}
m \\
k
\end{array}\right)_{r_{i} s_{i}^{-1}} c_{i j}^{(k)} \partial_{i}^{m-k} \partial_{j} \partial_{i}^{k} \\
& \quad+\delta_{\ell, i} r^{-\langle i, j\rangle} s^{\langle j, i\rangle} \sum_{k=0}^{m}(-1)^{k}\left(\begin{array}{c}
m \\
k
\end{array}\right)_{r_{i} s_{i}^{-1}}(m-k)_{r_{i}^{-1} s_{i}} c_{i j}^{(k)} r^{-k\langle i, i\rangle} s^{k\langle i, i\rangle} \partial_{i}^{m-k-1} \partial_{j} \partial_{i}^{k} \\
& \quad+\delta_{i, \ell} \sum_{k=0}^{m}(-1)^{k}\left(\begin{array}{c}
m \\
k
\end{array}\right)_{r_{i} s_{i}^{-1}}(k)_{r_{i}^{-1} s_{i}} c_{i j}^{(k)} \partial_{i}^{m-k} \partial_{j} \partial_{i}^{k-1} \\
& \quad+\delta_{\ell, j} \partial_{i}{ }^{m} \sum_{k=0}^{m}(-1)^{k}\left(\begin{array}{c}
m \\
k
\end{array}\right)_{r_{i} s_{i}^{-1}} c_{i j}^{(k)} r^{-k\langle j, i\rangle} s^{k\langle i, j\rangle} \\
&= S_{1}+S_{2}+S_{3}+S_{4}=S_{1},
\end{aligned}
$$

其中 $S_{2}=-S_{3}, S_{4}=0$ (由下面的引理 4.4), 以及

$$
\begin{aligned}
& S_{1}=r^{-m\langle\ell, i\rangle} s^{m\langle i, \ell\rangle} r^{-\langle\ell, j\rangle} s^{\langle j, \ell\rangle} E_{\ell} \sum_{k=0}^{m}(-1)^{k}\left(\begin{array}{c}
m \\
k
\end{array}\right)_{r_{i} s_{i}^{-1}} c_{i j}^{(k)} \partial_{i}^{m-k} \partial_{j} \partial_{i}^{k}, \\
& S_{2}=\delta_{\ell, i} \sum_{k=0}^{m-1}(-1)^{k}\left(\begin{array}{c}
m \\
k
\end{array}\right)_{r_{i} s_{i}^{-1}}(m-k)_{r_{i}^{-1} s_{i}} c_{i j}^{(k)} r^{-\langle i, j\rangle} s^{\langle j, i\rangle} r^{-k\langle i, i\rangle} s^{k\langle i, i\rangle} \partial_{i}^{m-k-1} \partial_{j} \partial_{i}^{k},
\end{aligned}
$$




$$
\begin{aligned}
& S_{3}=\delta_{i, \ell} \sum_{k=1}^{m}(-1)^{k}\left(\begin{array}{c}
m \\
k
\end{array}\right)_{r_{i} s_{i}^{-1}}(k)_{r_{i}^{-1} s_{i}} c_{i j}^{(k)} \partial_{i}{ }^{m-k} \partial_{j} \partial_{i}^{k-1}, \\
& S_{4}=\delta_{\ell, j} \partial_{i}{ }^{m} \sum_{k=0}^{m}(-1)^{k}\left(\begin{array}{c}
m \\
k
\end{array}\right)_{r_{i} s_{i}^{-1}} c_{i j}^{(k)} r^{-k\langle j, i\rangle} s^{k\langle i, j\rangle} .
\end{aligned}
$$

现在根据归纳假设, 得到 $S_{1} v=0$. 于是我们证明了等式 (**). 这意味着 $U_{r, s}^{+}$可藉由斜微 分算子 $\partial_{i}$ 来加以实现, 即由算子生成的代数是 $U_{r, s}^{+}$的一个同态像.

(ii) 类似地, 我们可证明另一个恒等式 (ii), 于是, $U_{r, s}^{+}$可以由斜微分算子 $\partial_{i}^{\prime}$ 来实现.

引理 4.4 (i) $S_{2}=-S_{3}$. (ii) $S_{4}=0$.

证明 (ii) 由下面的恒等式推出:

$$
\sum_{k=0}^{m}(-1)^{k}\left(\begin{array}{c}
m \\
k
\end{array}\right)_{r_{i} s_{i}^{-1}} c_{i j}^{(k)} r^{-k\langle j, i\rangle} s^{k\langle i, j\rangle}=\sum_{k=0}^{m}(-1)^{k}\left(\begin{array}{c}
m \\
k
\end{array}\right)_{r_{i} s_{i}^{-1}}\left(r_{i} s_{i}^{-1}\right)^{\frac{k(k-1)}{2}}=0 .
$$

至于 (i), 注意到 $(n)_{q^{-1}}=q^{1-n}(n)_{q}, 1-m=a_{i j}$, 以及

$$
\begin{aligned}
& c_{i j}^{(k-1)}=c_{i j}^{(k)} r^{-\langle j, i\rangle} s^{\langle i, j\rangle}\left(r_{i} s_{i}^{-1}\right)^{1-k}, \\
& r^{-\langle j, i\rangle-\langle i, j\rangle} s^{\langle i, j\rangle+\langle j, i\rangle}=\left(r_{i}^{-1} s_{i}\right)^{a_{i j}}=\left(r_{i} s_{i}^{-1}\right)^{m-1}, \\
&\left(\begin{array}{c}
m \\
k-1
\end{array}\right)_{r_{i} s_{i}^{-1}}(m-k+1)_{r_{i}^{-1} s_{i}}=\left(\begin{array}{c}
m \\
k-1
\end{array}\right)_{r_{i} s_{i}^{-1}}(m-k+1)_{r_{i} s_{i}^{-1}}\left(r_{i} s_{i}^{-1}\right)^{k-m} \\
&=\left(\begin{array}{c}
m \\
k
\end{array}\right)_{r_{i} s_{i}^{-1}}(k)_{r_{i} s_{i}^{-1}}\left(r_{i} s_{i}^{-1}\right)^{k-m} \\
&=\left(\begin{array}{c}
m \\
k
\end{array}\right)_{r_{i} s_{i}^{-1}}(k)_{r_{i}^{-1} s_{i}}\left(r_{i} s_{i}^{-1}\right)^{2 k-m-1},
\end{aligned}
$$

使得

$$
c_{i j}^{(k-1)} r^{-\langle i, j\rangle} s^{\langle j, i\rangle}\left(r_{i}^{-1} s_{i}\right)^{k-1}=c_{i j}^{(k)}\left(r_{i} s_{i}^{-1}\right)^{m-1+2(1-k)} .
$$

因而得到

$$
\begin{aligned}
S_{2}= & \delta_{\ell, i} \sum_{k=0}^{m-1}(-1)^{k}\left(\begin{array}{c}
m \\
k
\end{array}\right)_{r_{i} s_{i}^{-1}}(m-k)_{r_{i}^{-1} s_{i}} c_{i j}^{(k)} r^{-\langle i, j\rangle} s^{\langle j, i\rangle}\left(r_{i}^{-1} s_{i}\right)^{k} \partial_{i}^{m-k-1} \partial_{j} \partial_{i}^{k} \\
= & \delta_{\ell, i} \sum_{k=1}^{m}(-1)^{k+1}\left(\begin{array}{c}
m \\
k-1
\end{array}\right)_{r_{i} s_{i}^{-1}}(m-k+1)_{r_{i}^{-1} s_{i}} c_{i j}^{(k-1)} r^{-\langle i, j\rangle} s^{\langle j, i\rangle}\left(r_{i}^{-1} s_{i}\right)^{k-1} \\
& \times \partial_{i}^{m-k} \partial_{j} \partial_{i}^{k-1} \\
= & -\delta_{\ell, i} \sum_{k=1}^{m}(-1)^{k}\left(\begin{array}{c}
m \\
k
\end{array}\right)_{r_{i} s_{i}^{-1}}(k)_{r_{i}^{-1} s_{i}} c_{i j}^{(k)} \partial_{i}{ }^{m-k} \partial_{j} \partial_{i}^{k-1} \\
= & -S_{3} .
\end{aligned}
$$

证明完毕.

致谢胡乃红感谢 M. Rosso 和 V. K. Dobrev 对文献 $[9,10]$ 所作的有益评注 (由此而引发 了作者们对上圈形变问题的关注), 并感谢意大利国际理论物理研究中心 ICTP 对 他 2006 年在 ICTP 数学所访问的资助. 


\section{参考文献}

1 Artin M, Schelter W, Tate J. Quantum deformations of GL(n). Comm Pure Appl Math, 44: 879-895 (1991)

2 Reshetikhin N. Multiparameter quantum groups and twisted quasitriangular Hopf algebras. Lett Math Phys, 20: 331-335 (1990)

$3 \mathrm{Hu}$ N, Wang X. Quantizations of the generalized-Witt algebra and of Jacobson-Witt algebra in the modular case. J Algebra, 312: 902-929 (2007); arXiv.Math:QA/0602281

4 Benkart G, Witherspoon S. A Hopf structure for down-up algebras. Math Z, 238(3): 523-553 (2001)

5 Benkart G, Witherspoon S. Two-parameter quantum groups and Drinfel'd doubles. Algebr Represent Theory, 7: 261-286 (2004)

6 Benkart G, Witherspoon S. Representations of two-parameter quantum groups and Schur-Weyl duality. In: Hopf algebras, Lecture Notes in Pure and Appl Math, Vol 237. New York: Dekker, 2004, 65-92

7 Benkart G, Witherspoon S. Restricted two-parameter quantum groups. In: Fields Institute Communications. In: Representations of Finite Dimensional Algebras and Related Topics in Lie Theory and Geometry, vol 40. Providence: Amer Math Soc, 2004, 293-318

8 Benkart G, Kang S-J, Lee K H. On the center of two-parameter quantum groups. Proc Roy Soc Edingburg Sect A, 136(3): 445-472 (2006)

9 Bergeron N, Gao Y, Hu N. Drinfel'd doubles and Lusztig's symmetries of two-parameter quantum groups. J Algebra, 301: 378-405 2006; arXiv.Math:RT/0505614

10 Bergeron N, Gao Y, Hu N. Representations of two-parameter quantum orthogonal groups and symplectic groups. AMS/IP: Stud Adv Math, 39: 1-21 (2007); arXiv.Math:QA/0510124

$11 \mathrm{Hu} \mathrm{N}$, Shi Q. The two-parameter quantum group of exceptional type $G_{2}$ and Lusztig's symmetries. Pacific $J$ Math, 230(2): 327-346 (2007); arXiv.Math:QA/0601444

12 Bai X, Hu N. Two-parameter quantum groups of exceptional type $E$-series and convex PBW type basis. Algebr Colloq, 2008 (in press); arXiv.Math:QA/0605179

$13 \mathrm{Hu} \mathrm{N}$, Wang X. Restricted two-parameter quantum groups of type $B$ and their ribbon elements. Preprint, 2006 (42 pages)

14 王秀玲. Cartan 型 Lie 代数的量子化和限制 $B$ 型双参数量子群. 博士学位论文. 上海: 华东师范大学数 学系, 2007, 1-132

15 白晓棠. 双参数 $E$ 型量子群和限制 $D$ 型双参数量子群. 博士学位论文. 上海: 华东师范大学数学系, 2006, $1-84$

$16 \mathrm{Hu} \mathrm{N}$, Rosso M, Zhang H. Two-parameter affine quantum group $U_{r, s}\left(\widehat{\mathfrak{s} l_{n}}\right)$, Drinfel'd realization and quantum affine Lyndon basis. Comm Math Phys, 278: 453-486 (2008)

$17 \mathrm{Hu}$, Zhang $\mathrm{H}$. Vertex representations of two-parameter quantum affine algebras $U_{r, s}(\widehat{\mathfrak{g}})$ : The simply laced cases. Preprint 2006 (40 pages)

18 张红莲. 双参数量子仿射代数的 Drinfel'd 实现、量子仿射 Lyndon 基及其顶点表示. 博士学位论文. 上 海: 华东师范大学数学系, 2007, 1-148

19 裴玉峰. 多参数量子群的结构和实现. 博士学位论文. 上海: 华东师范大学数学系, 2007, 1-102

20 Kashiwara M. On crystal bases of the $q$-analogue of universal enveloping algebras. Duke Math J, 63: 465$516(1991)$ 